\title{
MEDICINA COMPLEMENTAR E ALTERNATIVA: UTILIZAÇÃO PELA COMUNIDADE DE MONTES CLAROS, MINAS GERAIS
}

\author{
Joao Felício Rodrigues Neto*1, Anderson Antônio de Faria², Maria Fernanda Santos Figueiredo ${ }^{3}$ \\ Trabalho desenvolvido na Universidade Estadual de Montes Claros - UNIMONTES, Centro de Ciências Biológicas e da Saúde - CCBS Departamento de Clínicas \\ Médicas Montes Claros, MG
}

\author{
*Correspondência: \\ Av.: Cula Mangabeira, 562 \\ Santo Expedito \\ CEP 39400-001 \\ Montes Claros, MG \\ Telefone: (38) 3224-8383 \\ Cel.(38)9961-3522 \\ Fax: (38) 3229-8284 \\ joao.felicio@unimontes.br
}

\begin{abstract}
RESUMO
OвJETIvo. Verificar a prevalência de utilização e o perfil socioeconômico do usuário de medicina complementar e alternativa pela população de Montes Claros (MG).

MÉTodos. Estudo transversal, analítico. A amostra foi probabilística, por conglomerados, sendo a unidade amostral o domicílio e os entrevistados de ambos os sexos e maiores de 18 anos. Os dados foram coletados em uma cidade de porte médio de Minas Gerais utilizando formulários semi-estruturados.

Resultados. Foram entrevistadas 3.090 pessoas. A prevalência de uso de medicina complementar e alternativas foi, quando consideradas somente as que envolvem custos, como homeopatia, acupuntura, quiropraxia, medicina ortomolecular, técnicas de relaxamento/meditação e massagem, de 8,93\% e $70 \%$, quando incluímos todas as terapias arguidas. As prevalências foram: oração a Deus (52\%), remédios populares (30,9\%), exercícios físicos $(25,5 \%)$, benzedeiras ( $15 \%)$, dietas populares $(7,1 \%)$, massagem $(4,9 \%)$, relaxamento/meditação $(2,8 \%)$, homeopatia $(2,4 \%)$, grupos de autoajuda $(1,9 \%)$, quiropraxia $(1,7 \%)$, acupuntura $(1,5 \%)$ e medicina ortomolecular $(0,2 \%)$. Mulheres, católicos, casados, melhor renda e escolaridade estiveram associados de forma positiva com a utilização das terapias que envolvem custos.

Conclusão. Medicina complementar e alternativa é utilizada por número significativo da população. Gênero, religião, estado civil, renda e escolaridade estiveram associados positivamente com utilização de terapias alternativas. 0 acesso das pessoas de menor renda e escolaridade à medicina complementar e alternativa poderia aumentar a prevalência de utilização daquelas formas que envolvem custos.
\end{abstract}

UnITERMos: Terapias complementares. Medicina alternativa. Medicina complementar. Homeopatia.

\section{INTRODUÇÃO}

Paralelamente à medicina oficialmente reconhecida, coexistem em nosso meio outras práticas de diagnóstico e de cuidados relacionados à saúde. A tendência na literatura é abrigá-las sob o termo medicina complementar e alternativa (MCA). Para o Centro Nacional de Medicina Complementar e Alternativa dos Estados Unidos (NCCAM), MCA "é o conjunto de diversos sistemas, práticas e produtos médicos e de atenção à saúde que não se consideram atualmente parte da medicina convencional" ${ }^{\prime}$. Medicina não convencional pode ser definida de forma funcional como sendo as intervenções que não são amplamente discutidas em escolas médicas e que geralmente não estão disponíveis nos hospitais ${ }^{2}$ ou que não estão em conformidade com os padrões da comunidade médica ${ }^{3}$.

A definição e o uso correto dos termos "complementar", alternativa" e "não convencional" são controversos, ${ }^{4,1}$ mas fogem ao escopo deste estudo e aqui eles serão usados como sinônimos.
Terapias médicas alternativas têm atraído cada vez mais atenção da mídia, da comunidade médica, dos órgãos governamentais e do público em geral ${ }^{5}$. Nos Estados Unidos, tem sido demonstrado sistematicamente o uso de medicina alternativa no cuidado à saúde ${ }^{5,6}$. Os gastos com profissionais de terapias alternativas aumentaram 45,2\% entre 1990 e 1997 nos Estados Unidos e foram estimados em U\$21,2 bilhões em 19976. Tratamentos não convencionais são usados por muitos médicos e por outros terapeutas por toda a Europa ${ }^{4}$, Austrália7 ${ }^{7}$ China $^{8}$ e Israel $^{9}$.

Os tipos de terapias alternativas utilizadas variam de um país para outro 4,10 . As formas mais comuns incluem: fitoterapia, massagem, homeopatia, oração a Deus, grupos de autoajuda, remédios populares, programas de dietas, acupuntura, quiropraxia, exercícios físicos, entre outros. O que é convencional num lugar, não o é, necessariamente, em outro. No Sul da África, existem 25.000 médicos alopatas e 200.000 curandeiros, de maneira que, pelo menos em número e acesso, o que é convencional para a maioria não é a medicina alopata

1. Professor Titular Departamento de Clínica Médica da Universidade Estadual de Montes Claros, Montes Claros, MG

2. Estudante de medicina da Universidade Estadual de Montes Claros, Montes Claros, MG

3. Enfermeira mestranda no Programa de Pós-graduação em Ciências da Saúde na Universidade Estadual de Montes Claros, Montes Claros, MG 
ocidental ${ }^{1}$. Ademais, em alguns países como China, ambas as Coréias e Vietnã, as medicinas complementar e alternativa não só coexistem com a medicina convencional como também estão integradas no Sistema de Saúde Pública ${ }^{1}$.

$\mathrm{O}$ aumento da popularidade da MCA reflete mudanças nas necessidades e valores na sociedade moderna em geral. Isto inclui um aumento nas doenças crônicas, aumento do acesso às informações em saúde e aumento da consciência de direito à qualidade de vida ${ }^{11}$.

Aproximadamente 1.500 artigos sobre MCA são publicados anualmente na literatura coberta pela MEDLINE ${ }^{12}$. Não obstante a esse aumento na demanda relatada no exterior, e a atenção atualmente dispensada à MCA, tanto na mídia quanto na comunidade científica, na América Latina existem poucas pesquisas que investiguem esse assunto de maneira sistemática ${ }^{13}$. No Brasil, só recentemente a MCA recebeu uma normatização do setor público, com a aprovação da Política Nacional de Práticas Integrativas e Complementares (PNPIC). ${ }^{14}$

A proposta deste trabalho foi determinar a prevalência de utilização de MCA na cidade de Montes Claros, Norte de Minas Gerais, Brasil; traçar o perfil socioeconômico do usuário; verificar se existe associação entre o sexo, nível de escolaridade, renda mensal e presença de comorbidades com a utilização de MCA. Além disso, pretende servir de fonte de informação para PNPIC.

\section{MÉtodos}

O trabalho foi conduzido dentro dos padrões exigidos pela declaração de Helsink e aprovada pelo Comitê de Ética em Pesquisa (CEP) da Universidade Estadual de Montes Claros.

O estudo é descritivo, transversal e a população alvo foi composta por pessoas residentes na cidade de Montes Claros, de ambos os sexos, acima de 18 anos. A amostragem foi probabilística, por conglomerados, dentro de extratos homogêneos, sendo a unidade amostral o domicílio.

O número total de pessoas maiores de 18 anos residentes na zona urbana de Montes Claros era de 224.451, segundo último recenseamento. A amostra foi calculada com base na prevalência esperada de $20 \%$ de utilização de medicina complementar e alternativa (MCA). Trabalhando com erro alfa de $2 \%$ e beta de 90\%, seriam necessárias cerca de 1.400 entrevistas para um estudo aleatório simples. Como o presente trabalho foi realizado por conglomerado, foi calculado o efeito do desenho da amostra $(\mathrm{DEF}=2,2)$, chegando-se a uma amostra total de 3.080 pessoas.

A amostra foi selecionada considerando os dados e conceitos do Instituto Brasileiro de Geografia e Estatística, IBGE, que divide a região de Montes Claros em 216 setores censitários. Não foram incluídos os setores não urbanos e urbanos especiais (asilos, creches, orfanatos, hospitais, conventos), que totalizavam 35 setores. Restaram 181 setores.

Numa segunda etapa, foi feito sorteio para determinar a ordem dos domicílios a serem visitados dentro de cada setor, sendo sorteado o número três. Então, os domicílios residenciais foram amostrados em ordem de três, e o primeiro de cada três foi visitado. Considerando que cada setor censitário tinha em média de 200 a 300 domicílios (e um em cada três seria visitado), foram sorteados 35 setores.
Quando não era possível a entrevista, por não haver domicílio residencial ou por não haver morador, o domicílio seguinte era visitado e a contagem reiniciada deste ponto.

Foram aplicados formulários semiestruturados (contendo 49 questões cada um), no período de Março a Agosto de 2005, por cinco estudantes de graduação em saúde previamente treinados para a tarefa. Durante duas semanas foram realizadas entrevistas-piloto e os questionários revisados. Após o início dos trabalhos, a cada semana os questionários eram recolhidos e verificados pelo coordenador de campo. Havendo falha na coleta ou preenchimento dos dados o entrevistador era notificado e o questionário excluído.

Para avaliar as características socioeconômicas e demográficas, os entrevistados foram questionados sobre idade, cor, situação conjugal, religião, atividade econômica, escolaridade e renda per capita familiar mensal.

Os participantes foram perguntados se já haviam feito uso de alguma terapia alternativa. As opções foram listadas aos entrevistados. As formas de terapias alternativas cujo uso foi investigado incluíram: acupuntura, homeopatia, massagem, programas de dietas, medicina ortomolecular, remédios populares, quiropraxia, exercícios físicos, benzedeiras, técnica de relaxamento/meditação, grupos de autoajuda, oração a Deus, guias espirituais, gurus e terreiros. A satisfação com o tratamento recebido também foi arguida.

A morbidade foi avaliada pelo relato do entrevistado, assim como o uso de medicamentos.

Foi utilizada a estatística descritiva para o enunciado dos dados. Para a comparação entre médias utilizou-se o teste T de Student e para comparações entre proporções, o teste do Qui quadrado. A significância estatística utilizada foi $p<0,05$.

\section{Resultados}

Foram visitados 3.117 domicílios, sendo que em 15 deles houve recusa à participação na pesquisa. Foram entrevistadas 3102 pessoas, mas $12(0,38 \%)$ questionários foram excluídos por falhas no preenchimento.

A prevalência de uso de medicina complementar e alternativa foi de $70 \%$, quando consideramos todos os tipos argüidos: oração a Deus com intenção terapêutica foi relatada por 1608 (52\%) dos entrevistados; remédios populares (30,9\%), exercícios físicos (25,5\%), benzedeiras (15\%), dietas populares $(7,1 \%)$, massagem $(4,9 \%)$, relaxamento/ meditação (2,8\%), homeopatia $(2,5 \%)$, grupos de autoajuda $(1,9 \%)$, quiropraxia $(1,7 \%)$, acupuntura $(1,5 \%)$ e medicina ortomolecular $(0,2 \%)$.

Quando consideramos "usuários" apenas aqueles que utilizaram formas terapêuticas que envolvem gastos com um profissional de saúde e/ou com a indústria farmacêutica, como homeopatia, acupuntura, quiropraxia, medicina ortomolecular, técnicas de relaxamento/meditação e massagem, a prevalência de uso caiu de 2.160 (70\%) para 276 (8,93\%). Nas comparações feitas a seguir, consideraremos "usuários" apenas aqueles que utilizaram as formas que envolvem gastos particulares e cujo uso, portanto, está influenciado pela disponibilidade de recursos financeiros.

As mulheres foram 1.936 (62,6\%). Elas utilizaram terapias alternativas com mais frequência que os homens $(p=0,00)$. A 
média de idade \pm desvio padrão foi de $42.05 \pm 16.13$ anos para os usuários de terapias alternativas e de $39,42 \pm 16.24$ para os não usuários. A comparação entre as faixas etárias não demonstrou prevalência de uso significativa de uma faixa específica sobre outra. $(p=0,17)$ (Tabela 1$)$.

As cores de pele mais frequentemente relatadas pelos entrevistados foram parda/mulata e branca e as menos comuns a negra e a amarela. Não houve diferença estatisticamente significativa de uso de MCA entre pessoas de cores diferentes neste trabalho. A religião mais professada foi a católica, seguida pela evangélica. A variável religião demonstrou maior diferença entre uso ou não-uso de MCA no grupo de "outras religiões" $(p=0,00)$. 0 estado civil esteve relacionado à utilização de MCA, sobretudo devido a diferença entre a categoria "divorciados" (Tabela 1 ).

Cerca de $32 \%$ dos que utilizaram MCA tinham renda superior a quatro salários mínimos e entre os não usuários este número foi de $15 \%$ ( $p=0,00)$. O número de pessoas com ensino superior completo foi cerca de cinco vezes maior entre os usuários de $\operatorname{MCA}(p=0,00)$.

Dos 3.090 entrevistados, $1.055(34,1 \%)$ relataram algum problema de saúde. Dentre eles, 144 haviam utilizado MCA (52,2\% dos usuários). Entre o grupo dos que não relataram problema de saúde $(\mathrm{N}=2035,65,9 \%)$ a utilização de $\mathrm{MCA}$ foi relatada por 132 pessoas (47,8\% dos usuários) $(p=0,00)$. Os principais problemas de saúde referidos foram: hipertensão arterial, por 279 dos entrevistados (9\%); lombalgia, 78 (2,5\%); gastrite, 59 (1,9\%); diabetes, 51 (1,6\%); sinusite, 31 (1\%); cardiopatia, 30 (0,9\%); cefaléia, 28 (0,9\%); bronquite, 26 $(0,8 \%)$; osteoporose, $23(0,7 \%)$, dentre outras.

Entre os usuários de MCA, $86(31,2 \%)$ consideravam sua saúde "excelente" ou "muito boa" e 94 (34,1\%) regular ou ruim. Entre os não usuários estes números foram 823 (29,2\%) e 816 (29\%), respectivamente $(p=0,94)$.

Os entrevistados foram perguntados se haviam recorrido a algum sistema de saúde nos últimos 12 meses. Dos entrevistados, 1.443 (46\%) haviam utilizado o serviço público de atenção à saúde nesse período; 931 (30,1\%) não havia utilizado nenhum serviço de saúde, enquanto 512 (16,6\%) recorreram a convênios e 294 (9,5\%) a serviços particulares nos 12 meses que antecederam a pesquisa.

O nível de satisfação com a medicina convencional foi questionado aos que foram submetidos a algum tratamento, tanto entre aqueles classificados como usuários de MCA quanto entre os não usuários. A comparação da satisfação com a medicina convencional entre esses grupos não demonstrou diferenças significativas.

\section{Discussão}

A utilização de medicina não convencional superou as estimativas. A prevalência de uso (considerando as formas que envolvem custos/consulta com profissional especializado) foi de $8,93 \%$ na população geral e de 13,6 \% entre os que relataram algum problema de saúde. Os índices de uso verificados superaram os relatados nos EUA ${ }^{6}$. Por um lado, a MCA pode ser a única solução para quem não tem acesso aos recursos da medicina moderna, o que pode chegar a $80 \%$ da população ${ }^{11}$. Neste caso as formas utilizadas geralmente não dependem de profissionais especializados e não incluem gastos com médicos e com a indústria farmacêutica, como oração a Deus, remédios populares, benzedeiras, dentre outros $(70 \%$ dos entrevistados em nossa amostra). De outro modo, sobretudo nos países desenvolvidos, a busca por terapias alternativas é uma opção para o tratamento de doenças crônicas como dor, câncer, portadores de HIV, ansiedade, depressão e insônia ${ }^{1}$.

O uso de terapias alternativas neste estudo foi influenciado pelo sexo, religião, estado civil, escolaridade e renda. Vários estudos indicam que usuários de MCA tendem a ser mulheres, pessoas de raça branca, de meia-idade, e com níveis educacionais mais elevados ${ }^{15}$. Neste estudo observou-se que as mulheres usaram formas terapêuticas não convencionas com mais frequência que os homens. Resultados semelhantes foram encontrados por Eisemberg 6 et al, em1997, em estudo realizado nos Estados Unidos, quando demonstraram que o uso era mais comum em mulheres (48,9\%) que em homens (37,8\%), e por Zollman ${ }^{16}$ no Reino Unido. 0 mesmo encontrado por estudos realizados em Singapura ${ }^{17}$ e Noruega ${ }^{16}$.

A idade dos entrevistados não teve associação estatisticamente significativa com o uso de MCA neste trabalho. No Reino Unido, a maioria dos usuários de MCA tem entre 35 e 60 anos $^{16}$. Pessoas com idade entre 35 e 49 anos utilizaram com maior frequência a MCA $(50,1 \%)$ que pessoas mais jovens $(41,8 \%)$ ou mais idosas $(39,1 \%)$ nos Estados Unidos ${ }^{6}$. Nos Países Escandinavos ${ }^{18}$ a maioria dos usuários tem entre 30 e 59 anos. 0 uso de terapias alternativas com maior frequência nas pessoas entre 30 e 60 anos nesses países pode ser parcialmente explicada pela maior independência financeira e a autonomia para escolha dos meios de cuidados com a saúde.

Comparando os usuários e os não usuários de MCA neste estudo, observou-se que a cor da pele, diferente dos resultados de trabalhos realizados em outros países, não esteve relacionada com a utilização de terapias alternativas. Nos Estados Unidos, o uso foi menos comum entre os descendentes de africanos $(33,1 \%)$ que entre outros grupos raciais $(44,5 \%)^{6}$. Esta associação entre a cor de pele e o uso de MCA pode ser explicada pelas diferenças socioeconômicas entre as etnias e pelas restrições de acesso a algumas formas terapêuticas decorrentes dos custos envolvidos nesses países. Em nosso meio, a miscigenação da população brasileira talvez possa justificar a inexistência de associação entre a cor e a utilização de MCA.

Renda e escolaridade mais elevadas têm sido sistematicamente demonstrado entre os usuários de MCA em relação aos não usuários ${ }^{6,16}$, o que também foi demonstrado neste estudo. O percentual de pessoas com renda mensal familiar acima de oito salários mínimos foi cerca de quatro vezes maior entre os usuários de MCA. Embora uma análise individual seja dificultada, a influência de cada um desses fatores - renda e escolaridade - é constatada de maneira independente nos estudos internacionais. Renda mensal elevada pode implicar mais informações sobre as opções da medicina não-convencional e também maior acesso aos serviços. Níveis mais elevados de escolaridade, isoladamente, contribuem precipuamente no fator informação.

O percentual de entrevistados com curso superior foi cerca de quatro vezes maior nos usuários de medicina complementar em relação aos não usuários. Nos Estados Unidos, o uso de MCA foi maior entre os que tinham curso superior $(50,6 \%)$ que 


\begin{tabular}{|c|c|c|c|c|c|}
\hline \multirow[t]{2}{*}{ Característica } & \multicolumn{2}{|c|}{$\begin{array}{l}\text { Não usuário } \\
\text { de MCA* }\end{array}$} & \multicolumn{2}{|c|}{$\begin{array}{l}\text { Usuário } \\
\text { de MCA }\end{array}$} & \multirow{2}{*}{$\begin{array}{c}\text { Valor } \\
\mathrm{p}\end{array}$} \\
\hline & $\mathrm{N}$ & $\%$ & $\mathrm{~N}$ & $\%$ & \\
\hline \multicolumn{6}{|l|}{ Renda } \\
\hline Ate um sm** & 503 & 17.8 & 25 & 9.1 & 0.00 \\
\hline De 1 a $2 \mathrm{sm}$ & 1033 & 36.7 & 78 & 28.3 & \\
\hline De 2 a $4 \mathrm{sm}$ & 872 & 31.0 & 85 & 30.7 & \\
\hline De 4 a 8 sm & 317 & 11.3 & 56 & 20.3 & \\
\hline De 8 a $16 \mathrm{sm}$ & 76 & 2.7 & 23 & 8.4 & \\
\hline Acima de $16 \mathrm{sm}$ & 13 & 0.5 & 9 & 3.2 & \\
\hline \multicolumn{6}{|l|}{ Escolaridade } \\
\hline Analfabeto & 209 & 7.4 & 16 & 5.7 & 0.00 \\
\hline $1^{0}$ grau inc. & 1206 & 42.9 & 74 & 26.8 & \\
\hline $1^{\circ}$ grau comp. & 424 & 15.1 & 27 & 9.7 & \\
\hline $2^{\circ}$ grau inc. & 99 & 3.5 & 6 & 2.2 & \\
\hline $2^{\circ}$ grau comp. & 687 & 24.4 & 89 & 32.3 & \\
\hline $3^{\circ}$ grau inc. & 34 & 1.2 & 6 & 2.2 & \\
\hline $3^{0}$ grau comp. & 155 & 5.5 & 58 & 21.1 & \\
\hline \multicolumn{6}{|l|}{ Sexo } \\
\hline Masculino & 1084 & 38.5 & 70 & 25.4 & 0.00 \\
\hline Feminino & 1730 & 61.5 & 206 & 74.6 & \\
\hline \multicolumn{6}{|l|}{ Religião } \\
\hline Católica & 2033 & 72.3 & 196 & 71.0 & 0.00 \\
\hline Evangélica & 654 & 23.2 & 48 & 17.4 & \\
\hline Outras & 21 & 0.7 & 14 & 5.1 & \\
\hline Não tem religiao & 106 & 3.8 & 18 & 6.5 & \\
\hline \multicolumn{6}{|l|}{ Estado conjugal } \\
\hline Solteiro & 970 & 34.5 & 92 & 33.3 & 0,00 \\
\hline Divorciado-separado & 159 & 5.6 & 29 & 10.5 & \\
\hline Viúvo & 197 & 7.0 & 22 & 8.0 & \\
\hline Casado & 1316 & 46.8 & 118 & 42.8 & \\
\hline União estável & 172 & 6.1 & 15 & 5.4 & \\
\hline \multicolumn{6}{|l|}{ Idade } \\
\hline De 18 a 30 anos & 1049 & 37.3 & 85 & 30.8 & 0.17 \\
\hline De 31 a 50 anos & 1060 & 37.7 & 111 & 40.27 & \\
\hline De 51 a 60 anos & 341 & 12.1 & 40 & 14.5 & \\
\hline Maiores de 60 anos & 364 & 12.9 & 40 & 14.5 & \\
\hline \multicolumn{6}{|l|}{ Cor } \\
\hline Parda ou mulata & 1234 & 43.9 & 109 & 39.5 & 0.10 \\
\hline Branca & 1232 & 43.8 & 143 & 51.9 & \\
\hline Negra & 297 & 10.5 & 20 & 7.2 & \\
\hline Amarela & 50 & 1.8 & 4 & 1.4 & \\
\hline Outras & 1 & 0 & 0 & 0 & \\
\hline
\end{tabular}

* MCA $=$ Medicina complementar e alternativa ** SM = Salário mínimo

entre os que não tinham $(36,4 \%)^{2}$. Essas diferenças de renda e escolaridade se acentuam com algumas formas específicas de terapias alternativas, provavelmente devido ao conhecimento sobre as diferentes alternativas disponíveis e à necessidade de consulta à profissional especializado, como a homeopatia.

A despeito do aumento geral no uso de MCA relatado nos estudos internacionais, $6,4,7$, o tipo específico de terapias não convencionais utilizadas em nosso meio variou consideravelmente. Remédios populares foram relatados por 30,9\% dos entrevistados. Este percentual foi 4,2\% nos Estados Unidos. Santos ${ }^{19}$ realizou um trabalho com professores e alunos do $1^{\circ}$ grau em Belo Horizonte e demonstrou que 67,5\% deles acreditavam que os remédios caseiros podem tratar qualquer doença e oração a Deus com finalidade terapêutica foi relatada por $82,2 \%$ dos alunos da primeira e segunda séries; $52 \%$ dos entrevistados do presente trabalho relataram oração a Deus com finalidade terapêutica. Um estudo feito por Harris ${ }^{20}$ et al., em 1999, sugeriu que a oração pode ser um efetivo auxílio aos cuidados médicos.

Quiropraxia, homeopatia, fitoterapia e acupuntura estão entre as mais populares terapias alternativas no Reino Unido ${ }^{16}$. Em relação ao uso de homeopatia, enquanto foi encontrado neste trabalho uma prevalência de $2,4 \%$, na Bélgica este percentual foi de $56 \%$; $32 \%$ na França, $28 \%$ na Dinamarca ${ }^{16}$ e 3,4\% nos Estados Unidos 6 . Uso de acupuntura foi relatado por 1,5\% dos nossos entrevistados. Nos Países Baixos esse número é de 16\%, semelhante à França $(21 \%)$ e Bélgica $(19 \%)^{16}$; nos EUA é de $1 \%{ }^{6}$. Entretanto, neste estudo, a frequência de utilização da homeopatia e outras formas terapêuticas poderia ser maior caso a MCA estivesse disponível nos serviços públicos, pois cerca de $50 \%$ dos que recorram aos serviços de saúde nos últimos 12 meses o fez nesses serviços. Formas terapêuticas que envolvem manipulação, como quiropraxia e osteopatia, têm prevalência de $48 \%$ na Suécia e $36 \%$ no Reino Unido ${ }^{4}$. O uso de quiropraxia encontrado neste estudo foi 1,7\% (Gráfico 1).

A presença de doenças crônicas esteve associada com a maior procura por terapias não convencionais neste trabalho. Estudos quantitativos e qualitativos mostram que pessoas que consultam com terapeutas de medicina complementar normalmente têm condições crônicas para as quais a medicina tradicional não forneceu solução satisfatória 7 . O uso de MCA é popular entre pacientes com doenças crônicas como câncer, AIDS, artrite, asma, Diabetes mellitus, epilepsia, etc. ${ }^{11}$. Na Alemanha, $77 \%$ das clínicas de dor usam acupuntura ${ }^{21}$.

$\mathrm{O}$ uso de MCA para medidas preventivas também merece ser destacado. Em 1997, nos EUA, 42\% de todas as terapias alternativas foram utilizadas para tratar alguma doença, enquanto $58 \%$ foram usadas, pelo menos em parte, para prevenir doenças futuras ou manter a saúde e vitalidade ${ }^{6}$.

Por todo mundo, um grande número de pacientes está saindo da medicina convencional para procurar ajuda ${ }^{11}$. 0 aumento na demanda foi observado em vários países citados neste artigo e de uma maneira geral pode se dizer que a crescente popularidade da medicina complementar e alternativa é um fato mundial.

O rápido aumento do interesse público e do uso de terapias alternativas e complementares está exercendo uma forte influência na educação médica. A maioria das escolas médicas dos Estados Unidos agora oferece cursos de MCA ${ }^{6}$. Não obstante, a maioria das escolas médicas brasileiras não dispõe em seu currículo de um espaço para discussão deste assunto. Em São Paulo, Teixeira ${ }^{22}$ et al. avaliaram o ensino de práticas não convencionais nas escolas de medicina e o interesse de estudantes e médicos sobre terapias alternativas. Eles constataram que, com o aumento da procura de práticas não convencionais os médicos estão começando a sentir a necessidade de suprir esta demanda. Numa amostra de 484 estudantes da Faculdade de Medicina da Universidade de São Paulo, este mesmo trabalho demonstrou que $85 \%$ deles consideravam que homeopatia e acupuntura 
deveriam estar inseridas no currículo da graduação das escolas médicas, de forma opcional (72\%) ou obrigatória (18\%).

Pesquisas com usuários de medicina complementar indicam que cerca de $80 \%$ estão satisfeitos com o tratamento que receberam $^{11}$. 0 crescimento da medicina alternativa, mesmo sendo uma realidade cada vez mais presente nos serviços de saúde, apresenta grandes desafios para sua institucionalização, tais como: reduzido número de recursos humanos capacitados; insuficiente financiamento para a maioria das práticas; e poucos espaços institucionais para seu desenvolvimento nos serviços. ${ }^{23}$

Considerando a escassez de informações sobre MCA em nosso meio, observamos neste estudo relevantes informações que podem contribuir para a definição de políticas públicas de saúde para a população brasileira. Observamos, com as informações deste estudo, que em Montes Claros, a universalidade, um dos princípios constitucionais do Sistema Único de Saúde - SUS, em relação à MCA não está sendo atendida. Pois o acesso e uso da MCA têm frequência maior em pessoas economicamente ativas, com maior nível de renda e escolaridade, com acesso aos planos de saúde ou serviços particulares, sendo dificultado àqueles que dispõem somente dos serviços públicos de saúde. Entretanto, a aprovação da PNPIC demonstra a coerência do SUS com seus princípios. Além disso, é preciso que as instituições de ensino em saúde devam assumir uma atitude pró-ativa, estar atentas às necessidades de saúde da população, empenhando-se na formação na graduação, pós graduação, educação permanente e continuada de profissionais capacitados à prática de MCA.

\section{Conclusão}

Este estudo nos permitiu conhecer a prevalência de uso da medicina complementar e alternativa, além de traçar o perfil do seu usuário na cidade de Montes Claros, Minas Gerais. Os resultados deste trabalho são importantes por fornecer as primeiras informações epidemiológicas de base populacional no Brasil. Um número significativo de pessoas recorreu à MCA, principalmente aquelas com maiores renda e escolaridade. As possíveis diferenças com outras regiões do Brasil quanto ao uso de MCA e os resultados aqui encontrados demonstram a necessidade de esforços e ações para realização de um estudo multicêntrico no país, visando contribuir para a política nacional de práticas integrativas e complementares.

\section{Agradecimentos}

1 - Ao CNPq pelo financiamento para o Projeto de Pesquisa: Medicina Complementar e Alternativa: conhecimento e utilização da Homeopatia pela comunidade de Montes Claros, Norte de Minas Gerais.

2 - À Marise Fagundes Silveira, pela contribuição na análise estatística do trabalho.

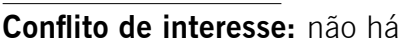

\section{SUMmARY}

Complementary and alternative medicine: use in Montes Claros, Minas Gerais

OBJECTIVE. To determine prevalence of utilization and social and economic profile of those using complementary and alternative medicine in the medium sized Brazilian city of Montes Claros, $M G$.

Methods. A transversal descriptive study was conducted. The sample of 3090 people was probabilistic, by clusters using the household as the sample unit for interview of both genders, older than 18 years. Data were collected by semi-structured questionnaires.

RESULTS. Utilization of complementary and alternative medicine was of $8.9 \%$ when only those involving costs such as homeopathy, acupuncture, chiropractics, techniques of relaxation/ meditation and massage are considered and of $70.0 \%$, when all therapies found were included. Prevalent were prayers to God (52.0\%), popular medicines (30.9\%), physical exercises (25.5\%), faith healers (15.0\%), popular diets (7.1\%), massage (4.9\%), relaxation/meditation (2.8\%), homeopathy (2.4\%), and groups of self-help (1.9\%), chiropractics (1.7\%), acupuncture (1.5\%) and orthomolecular medicine (0.2\%). Women, Catholic, married of higher income and education were positively associated with utilization of therapies involving expenses.

Conclusions. Complementary and alternative medicine is used by a significant number of those interviewed. Gender, religion, marital status, income and education were positively associated with utilization of complementary and alternative medicine. Access of those with less income and education could increase the utilization of the options that involve expenses. [Rev Assoc Med Bras 2009; 55(3): 296-301]

KEY wORDS: Complementary therapies. Complementary therapies. Complementary medicine and homeopathy.

\section{REFERÊNCIAS}

1. Nogales-Gaete J. Medicina alternativa y complementaria. Rev Chil NeuroPsiquiatr. 2004; 42:243-50.

2. Eisemberg DM, Kessler RC, Foster C, Norlock FE, Calkins DR, Delbanco TL. Unconventional medicine in the United States. N Engl J Med. 1993;328:246-52

3. Gevitz N. Three perspectives on unorthodox medicine. In: Gevitz N, editor. Other healers: unorthodox medicine in America. Baltimore: John Hopkins University Press; 1998. p.1-28

4. Fisher P, Ward A. Complementary medicine in Europe. BMJ. 1994; 309:107-11.

5. Eisemberg DM. Advising patients who seek alternative medical therapies. Ann Intern Med. 1997;127: 61-9.

6. Eisemberg DM, Davis RB, Ettner SL, Appel S, Wilkey S, Rompay MV, et al. Trends in Alternative Medicine use in the United States, 1990-1997: results of a follow-up national survey. JAMA. 1998; 280: 1569-75.

7. Maclennan AH, Wilson DH, Taylor AW. Prevalence and cost of alternative medicine in Australia. Lancet. 1996; 347:569-73.

8. Ergil KV. In: Micozzi F, editor. Foundamentals of complementary and alternative medicine. New York (NY): Livingstone; 1996. p.185-223. In: Pal SK. Complementary and alternative medicine : an overview. Curr Sci. 2002;82:518-24.

9. Ernest E, Sier-Ner I, Gamus D. Complementary medicine: a review of immunomodulatory effects of chinese herbal medicine. Isr J Med Sci. 1997;33:808-15.

10. Grossman E. Complementary and alternative medicine: the facts. Isr Med Assoc J. 2005;7:602-3.

11. Pal SK. Complementary and alternative medicine: an overview. Curr Sci. 2002; 82: 518-24.

12. Ernest E. Prevalence of use of complementary medicine: a systematic review. Bull World Health Organ. 2000;78:252-7.

13. Barros NF, Nunes ED. Complementary and alternative medicine in Brazil: one concept, different meanings. Cad Saúde Pública 2006; 22:2023-9.

14. Brasil Ministério da Saúde. Portaria No 971, 03 de maio de 2006. 
15. Lee GBW, Charn ZH, Ng TP. Complementary and alternativemedicine usi in patients with chronic diseases in primary care is associated with perceived quality of care and cultural beliefs. Fam Pract. 2004; 21:1-6.

16. Zollman $\mathrm{C}$, Vickers $\mathrm{A}$. ABC of complementary medicine. Users and practitioners of complementary medicine. BMJ. 1999;319:836-8.

17. Lim MK, Sadarangani P, Chan HL, Heng JY. Complementary and alternative medicine use in multiracial Singapore. Complement Ther Med. 2005; 13:16-24.

18. Hanssen B, Grimsgaard S, Launso L, Fonebo V, falkenberg T, Rasmussem NKR. Use of complementary and alternative medicine in the Scandinavian countries. Scand J Prim Health Care. 2005; 23: 57-62.

19. Santos MG, Dias AGP, Martins MM. Conhecimento e uso da medicina alternativa entre alunos e professores de primeiro grau. Rev Saúde Pública. 1995;29:221-7.

20. Harris WS, Gowda M, Kolb JW, Strychacz CP, Vacek JL, Jones PG, et al. A randomized, controlled trial of the effects of remote, intercessory prayer on outcomes in patients admitted to the coronary care unit. Arch Intern Med 1999;159: 2273-8.

21. Marthiessen P, Rosslenbroich B, Schmidt St. Unkonventionelle Medizinische Richtungen-Bestandaufnahme zur froschungssituation. apud Fisher $\mathrm{P}$, Ward A. Complementary medicine in Europe. BMJ. 1994;309: 107-11.

22. Teixeira MZ, Lin CA, Martins MA. O ensino de práticas não convencionais em saúde nas faculdades de medicina: panorama Mundial e perspectivas brasileiras Rev Bras Educ Méd. 2004; 28:51-9.

23. Souza IMC, Vieira ALS. Serviços públicos de saúde e medicina alternativa. Cienc Saúde Coletiva. 2005;10(Supl): 255-66.

Artigo recebido: 08/01/08

Aceito para publicação: 01/10/08 